\title{
Supplemental materials for "On the choice of difference sequence in a unified framework for variance estimation in nonparametric regression"
}

\author{
Wenlin Dai ${ }^{1}$, Tiejun Tong ${ }^{2}$ and Lixing $\mathrm{Zhu}^{2}$ \\ ${ }^{1}$ King Abdullah University of Science and Technology, Thuwal 23955-6900, Saudi Arabia \\ ${ }^{2}$ Department of Mathematics, Hong Kong Baptist University, Hong Kong
}

This document serves as a supplement to the main manuscript and is organized as follows. In Section S1, we describe in detail the proposed unified framework for estimating the residual variance. In Section S2, we derive the asymptotic results of the unified variance estimators. In Section S3, we conduct a numerical comparison on the bias terms of the unified estimators with the "state-of-the-art" estimator in the literature. In Section S4, we provide an alternative procedure for the unified estimation for unequally spaced design. Proofs of theoretical results are provided in Section S5.

\section{S1 Unified framework}

We consider the nonparametric regression model

$$
Y_{i}=g\left(x_{i}\right)+\varepsilon_{i}, \quad i=1, \ldots, n,
$$

where $\left\{Y_{i}\right\}$ are the observations, $g$ is an unknown mean function, $\left\{x_{i}\right\}$ are the design points, and $\left\{\varepsilon_{i}\right\}$ are the independent and identically distributed (i.i.d.) random errors with mean zero and variance $\sigma^{2}$.

For the difference-based variance estimation, two popular methods in the literature are the higher-order difference estimator in Hall, Kay and Titterington (1990) and the linear regression estimator in Tong and Wang (2005). To combine the two methods 
systematically, for any order- $r$ difference sequence $d=\left(d_{0}, \ldots, d_{r}\right)$ satisfying

$$
\sum_{j=0}^{r} d_{j}=0 \quad \text { and } \quad \sum_{j=0}^{r} d_{j}^{2}=1
$$

we define

$$
s_{k}(r)=\frac{1}{n-r k} \sum_{i=1}^{n-r k}\left(\sum_{j=0}^{r} d_{j} Y_{i+j k}\right)^{2} .
$$

Let $J(r)=\left(\sum_{j=0}^{r} j d_{j}\right)^{2} \int_{0}^{1}\left[g^{\prime}(x)\right]^{2} d x, \alpha=\sigma^{2}, \beta=J(r)$ and $h_{k}=k^{2} / n^{2}$, we have the approximately linear regression model $s_{k}(r) \approx \alpha+h_{k} \beta$. Then we fit the linear regression model by minimizing the following weighted sum of squares

$$
\sum_{k=1}^{m} w_{k}\left(s_{k}(r)-\alpha-h_{k} \beta\right)^{2}, \quad \beta>0
$$

where $w_{k}=(n-r k) / N$ are the corresponding weights with $N=\sum_{k=1}^{m}(n-r k)=n m-$ $r m(m+1) / 2$. Finally, we estimate $\sigma^{2}$ by the fitted intercept in the unified framework. This leads to the unified estimator as

$$
\hat{\sigma}^{2}(r, m)=\hat{\alpha}=\sum_{k=1}^{m} b_{k} w_{k} s_{k}(r),
$$

where $b_{k}=1-\bar{h}_{w}\left(h_{k}-\bar{h}_{w}\right) /\left(\sum_{k=1}^{m} w_{k} h_{k}^{2}-\bar{h}_{w}^{2}\right)$ and $\bar{h}_{w}=\sum_{k=1}^{m} w_{k} h_{k}$. Further, if the optimal difference sequence is used in (S2), we refer to the unified estimator as the unified optimal estimator, denoted by $\hat{\sigma}_{\text {opt }}^{2}(r, m)$. Otherwise if the ordinary difference sequence is used, we refer to it as the unified ordinary estimator, denoted by $\hat{\sigma}_{\text {ord }}^{2}(r, m)$.

Theorem S1. For the equally spaced design, we have (i) the proposed estimator $\hat{\sigma}^{2}(r, m)$ is an unbiased estimator for any difference sequence satisfying (S1) when $g(x)$ is a linear function; and (ii) for the ordinary difference sequence, the estimator is an unbiased estimator for any polynomial $g(x)$ with order up to $p \leq r-1$.

The proof of Theorem S1 is given in Section S5. Theorem S1 shows that the linear regression does provide merit in correcting the estimation bias, especially when the mean function is not very rough. 


\section{S2 Asymptotic results}

We first derive the asymptotic bias of the unified estimator (S3). To cater for the demand of difference sequence selection, we also specify the relevant results for the optimal difference sequence and ordinary difference sequence, respectively. By the following two theorems, we observe that the unified estimator $\hat{\sigma}^{2}(r, m)$ is capable to control the bias to order $O\left(\mathrm{~m}^{3} / \mathrm{n}^{3}\right)$ for the optimal difference sequence, and to order $O\left(\mathrm{~m}^{2 r} / \mathrm{n}^{2 r}\right)$ for the ordinary difference sequence. This demonstrates that the linear regression with the ordinary difference sequence provides a smaller asymptotic bias than the linear regression with the optimal difference sequence for any $r \geq 2$.

Theorem S2. Consider the equally spaced design with $m \rightarrow \infty$ and $m / n \rightarrow 0$, and assume that $g(x)$ has a bounded second derivative. Then for any difference sequence $\left(d_{0}, \ldots, d_{r}\right)$ under the constraint (S1) with $r \geq 1$,

$E\left(\hat{\sigma}^{2}(r, m)\right)=\sigma^{2}-\frac{C_{1}}{16}\left[r C_{1} \int_{0}^{1}\left[g^{\prime}(t)\right]^{2} d t-\left(r C_{1}-C_{2}\right)\left[g^{\prime}(1)\right]^{2}-C_{2}\left[g^{\prime}(0)\right]^{2}\right] \frac{m^{3}}{n^{3}}+o\left(\frac{m^{3}}{n^{3}}\right)$,

where $C_{i}=\sum_{j=0}^{r} j^{i} d_{j} / i$ ! for $i=1$ and 2 .

Theorem S3. Consider the equally spaced design with $m \rightarrow \infty$ and $m / n \rightarrow 0$, and assume that $g(x)$ has a bounded rth derivative. Then for the ordinary difference sequence with $r \geq 2$,

$$
E\left(\hat{\sigma}_{\text {ord }}^{2}(r, m)\right)=\sigma^{2}+\left[\frac{3(1-r)}{(2 r+1)(2 r+3)\left(\begin{array}{c}
2 r \\
r
\end{array}\right)} \int_{0}^{1}\left[g^{(r)}(x)\right]^{2} d x\right] \frac{m^{2 r}}{n^{2 r}}+o\left(\frac{m^{2 r}}{n^{2 r}}\right) .
$$

Now we investigate the asymptotic variance of the unified estimator. Recall that an order of $r \geq 3$ is rarely recommended for practical use even for $m=1$ (Dette, Munk and Wagner 1998). Hence, for the sake of brevity, we present the asymptotic variance of the unified estimator only for $r=2$, i.e., the minimum order that distinguishes the optimal difference sequence from the ordinary difference sequence. Using a higher order with $r \geq 3$ will yield more tedious derivation and more complex solutions, yet the comparison results 
remain the same. With $r=2$, the unified estimator can be represented as

$$
\hat{\sigma}^{2}(2, m)=\sum_{k=1}^{m} b_{k} w_{k} s_{k}(2)=Y^{T} D Y / \operatorname{tr}(D),
$$

where $Y=\left(Y_{1}, \ldots, Y_{n}\right)^{T}, \operatorname{tr}(\cdot)$ is the trace, and $D=\left(d_{i j}\right)_{n \times n}$ is an $n \times n$ matrix with the diagonal elements

$$
d_{i i}=d_{0}^{2} \sum_{k=1}^{\min \left(m,\left[\frac{n-i}{2}\right]\right)} b_{k}+d_{1}^{2} \sum_{k=1}^{\min (m, n-i, i-1)} b_{k}+d_{2}^{2} \sum_{k=1}^{\min \left(m,\left[\frac{i-1}{2}\right]\right)} b_{k}, \quad i=1, \ldots, n,
$$

and the off-diagonal elements

$d_{i j}= \begin{cases}d_{0} d_{1} b_{k} I_{(1 \leq k \leq m)}+d_{0} d_{2} b_{k / 2} I_{(k \in E)} & 1 \leq|i-j|=k \leq 2 m, 1 \leq \min (i, j) \leq k, \\ -d_{1}^{2} b_{k} I_{(1 \leq k \leq m)}+d_{0} d_{2} b_{k / 2} I_{(k \in E)} & 1 \leq|i-j|=k \leq 2 m, k+1 \leq i, j \leq n-k, \\ d_{1} d_{2} b_{k} I_{(1 \leq k \leq m)}+d_{0} d_{2} b_{k / 2} I_{(k \in E)} & 1 \leq|i-j|=k \leq 2 m, n-k+1 \leq \max (i, j) \leq n, \\ 0 & \text { otherwise. }\end{cases}$

Here, $E$ is the set of positive even integers, $I$ is the indicator function, and $[x]$ refers to the largest integer smaller than or equal to $x$. We also let $\sum_{k=1}^{0} b_{k}=0$ for ease of notation.

To derive the asymptotic variance of $\hat{\sigma}^{2}(2, m)$ with the quadratic form $(\mathrm{S} 4)$, we apply the following formula:

$$
\begin{aligned}
\operatorname{var}\left(\hat{\sigma}^{2}(2, m)\right)=\frac{1}{\operatorname{tr}(D)^{2}}[ & 4 \sigma^{2} g^{T} D^{2} g+4 g^{T}(D \operatorname{diag}(D) u) \sigma^{3} \gamma_{3} \\
& \left.+\sigma^{4} \operatorname{tr}\left\{\operatorname{diag}(D)^{2}\right\}\left(\gamma_{4}-3\right)+2 \sigma^{4} \operatorname{tr}\left(D^{2}\right)\right]
\end{aligned}
$$

where $g=\left(g\left(x_{1}\right), \ldots, g\left(x_{n}\right)\right)^{T}, u=(1, \ldots, 1)^{T}$, $\operatorname{diag}(D)$ denotes the diagonal matrix of $D$, and $\gamma_{i}=E\left(\varepsilon^{i} / \sigma^{i}\right)$ for $i=3$ and 4 . Consequently, we show that

Theorem S4. Consider the equally spaced design with $m \rightarrow \infty$ and $m / n \rightarrow 0$, and assume that $g(x)$ has a bounded second derivative. Then for any difference sequence $\left(d_{0}, d_{1}, d_{2}\right)$ under the constraint (S1), we have

$$
\operatorname{var}\left(\hat{\sigma}^{2}(2, m)\right)=\frac{1}{n} \operatorname{var}\left(\varepsilon^{2}\right)+\frac{A_{1}}{m n} \sigma^{4}+\frac{A_{2} m}{n^{2}} \operatorname{var}\left(\varepsilon^{2}\right)+o\left(\frac{1}{n m}\right)+o\left(\frac{m}{n^{2}}\right),
$$

where $A_{1}=9 / 4+9 d_{1}^{2}\left(d_{1}^{2}-1 / 2\right)$ and $A_{2}=9 / 56+165 d_{1}^{2}\left(1-d_{1}^{2}\right) / 448$.

By Theorems S2 and S4, the optimal bandwidth is $m_{\mathrm{opt}}=\sqrt{A_{1} \sigma^{4} /\left(A_{2} \operatorname{var}\left(\varepsilon^{2}\right)\right)} n^{1 / 2}$, and the optimal MSE is $\operatorname{MSE}\left(\hat{\sigma}^{2}\left(2, m_{\text {opt }}\right)\right)=n^{-1} \operatorname{var}\left(\varepsilon^{2}\right)+2 n^{-3 / 2} \sqrt{A_{1} A_{2} \sigma^{4} \operatorname{var}\left(\varepsilon^{2}\right)}+$ 
$o\left(n^{-3 / 2}\right)+O\left(m^{6} / n^{6}\right)$. In addition, we have $\operatorname{MSE}\left(\hat{\sigma}^{2}(2, m)\right)=n^{-1}\left[\operatorname{var}\left(\varepsilon^{2}\right)+o(1)\right]$ for any $m=n^{\tau}$ with $0<\tau<5 / 6$. This shows that the unified estimator can achieve the asymptotically optimal rate of MSE and hence is a consistent estimator of $\sigma^{2}$, no matter which difference sequence is used. From this point of view, the unified estimator has improved the classical difference-based estimators in Hall et al. (1990). Note also that, by a similar proof as that for Theorem $\mathrm{S} 4$, we can verify that $\operatorname{var}\left(\hat{\sigma}^{2}(r, m)\right)=n^{-1}\left[\operatorname{var}\left(\varepsilon^{2}\right)+o(1)\right]$ for any difference sequence of order $r \geq 3$ with $m \rightarrow \infty$ and $m / n \rightarrow 0$.

\section{S3 Bias comparison}

In this section, we conduct a numerical comparison on the bias terms of the "state-ofthe-art" estimator and the proposed unified estimators, as a supplement to Section 3 in the main manuscript. Specifically, we consider the TW estimator in Tong and Wang (2005), the unified ordinary estimator, and the unified optimal estimator. For simplicity

of exposition, we fix $r=2$ (the comparison results for $r \geq 3$ remain similar) and assume the errors are normally distributed. According to Theorems S1 and S2, we have

$$
\begin{aligned}
\operatorname{Bias}\left(\hat{\sigma}_{\mathrm{TW}}^{2}\right) & \simeq-\frac{1}{32}\left[\int_{0}^{1}\left[g^{\prime}(t)\right]^{2} d t-\frac{1}{2}\left[g^{\prime}(0)\right]^{2}-\frac{1}{2}\left[g^{\prime}(1)\right]^{2}\right] \frac{m^{3}}{n^{3}} \\
\operatorname{Bias}\left(\hat{\sigma}_{\text {opt }}^{2}(2, m)\right) & \simeq-\frac{5}{32}\left[\int_{0}^{1}\left[g^{\prime}(t)\right]^{2} d t-\frac{10-\sqrt{5}}{20}\left[g^{\prime}(0)\right]^{2}-\frac{10+\sqrt{5}}{20}\left[g^{\prime}(1)\right]^{2}\right] \frac{m^{3}}{n^{3}}, \\
\operatorname{Bias}\left(\hat{\sigma}_{\text {ord }}^{2}(2, m)\right) & \simeq-\left[\frac{1}{70} \int_{0}^{1}\left[g^{\prime \prime}(x)\right]^{2} d x\right] \frac{m^{4}}{n^{4}}
\end{aligned}
$$

As mentioned, the asymptotic bias of the unified ordinary estimator has a lower order than those of the TW estimator and the unified optimal estimator.

To numerically compare the coefficients in the bias terms, we consider the regression function $g(x)=A \sin (w \pi x)$, where $A$ represents the amplitude and $w$ represents the frequency. Note that the sine function has been frequently used in the literature for conducting simulation studies, see for example, in Seifert, Gasser and Wolf (1993), Dette et al. (1998), Tong and Wang (2005), and Tong, Ma and Wang (2013). Given the sine function, we have $\operatorname{Bias}\left(\hat{\sigma}_{\mathrm{TW}}^{2}\right) \simeq B_{1} m^{3} / n^{3}$, Bias $\left(\hat{\sigma}_{\text {opt }}^{2}(2, m)\right) \simeq B_{2} m^{3} / n^{3}$, and 
$\operatorname{Bias}\left(\hat{\sigma}_{\text {ord }}^{2}(2, m)\right) \simeq B_{3} m^{4} / n^{4}$, where the coefficients of the leading terms are

$$
\begin{aligned}
& B_{1}=-\frac{1}{64} w^{2} \pi^{2} A^{2}\left[\frac{\sin (w \pi) \cos (w \pi)}{w \pi}-\frac{1}{2}-\frac{1}{2} \cos (2 w \pi)\right], \\
& B_{2}=-\frac{5}{64} w^{2} \pi^{2} A^{2}\left[\frac{\sin (w \pi) \cos (w \pi)}{w \pi}-\frac{10-\sqrt{5}}{20}-\frac{10+\sqrt{5}}{20} \cos (2 w \pi)\right], \\
& B_{3}=-\frac{1}{140} w^{4} \pi^{4} A^{2}\left[1-\frac{\sin (w \pi) \cos (w \pi)}{w \pi}\right] .
\end{aligned}
$$

First of all, as the three coefficients are all proportional to $A^{2}$, we conclude that the amplitude $A$ takes no effect on the comparison of the estimators. The effect of $w$ on the corresponding bias terms is investigated in the next paragraph.

To compare the bias terms of the TW estimator and the unified optimal estimator, it suffices to compare the coefficients $B_{1}$ and $B_{2}$. With $w$ ranging from 0 to 4.1 , we plot in Figure $\mathrm{S} 1$ the quantites of $\left|B_{1}\right|$ and $\left|B_{2}\right|$ for $g(x)=5 \sin (w \pi x)$. It is evident that both $\left|B_{1}\right|$ and $\left|B_{2}\right|$ have a periodic pattern along with $w$, and $\left|B_{1}\right|<\left|B_{2}\right|$ for most $w$ values. In particular, we have $\left|B_{1}\right|=3.86$ and $\left|B_{2}\right|=19.28$ for $w=1,\left|B_{1}\right|=15.42$ and $\left|B_{2}\right|=77.11$ for $w=2$, and $\left|B_{1}\right|=61.69$ and $\left|B_{2}\right|=308.43$ for $w=4$. This shows that the bias term of the unified optimal estimator is even more severe than that of the TW estimator. In view of this, we conclude that a combination between the linear regression method and the optimal difference sequence can be less satisfactory. For the unified ordinary estimator, instead, it is always asymptotically better than the TW estimator as it is a higher order bias term, regardless of the comparison of coefficients.

\section{S4 Unequally spaced design}

For a general domain including the unequally spaced design, we provide an alternative procedure for the unified framework of estimating the residual variance. Assume that $\left\{x_{1}, \ldots, x_{n}\right\}$ are ordered design points with $x_{1}<\cdots<x_{n}$, and let $\mathcal{A}_{r}=\left\{(i, k): x_{i+k r}-\right.$ $\left.x_{i} \leq M, i+k r \leq n\right\}$ for $M=o(1)$. We define $p_{i k}=\left(\sum_{j=0}^{r} a_{i k j} Y_{i+k j}\right)^{2}$, where $\left\{a_{i k j}\right\}$ is

a difference sequence with order $r$. Then, $E\left(p_{i k}\right)=\sigma^{2}+q_{i k}^{2}\left[g^{\prime}\left(x_{i}\right)\right]^{2}+o\left(q_{i k}^{2}\right)$ where $q_{i k}=$ $\sum_{j=1}^{r} a_{i k j}\left(x_{i+k j}-x_{i}\right)$. With this approximation, we can construct the linear regression 


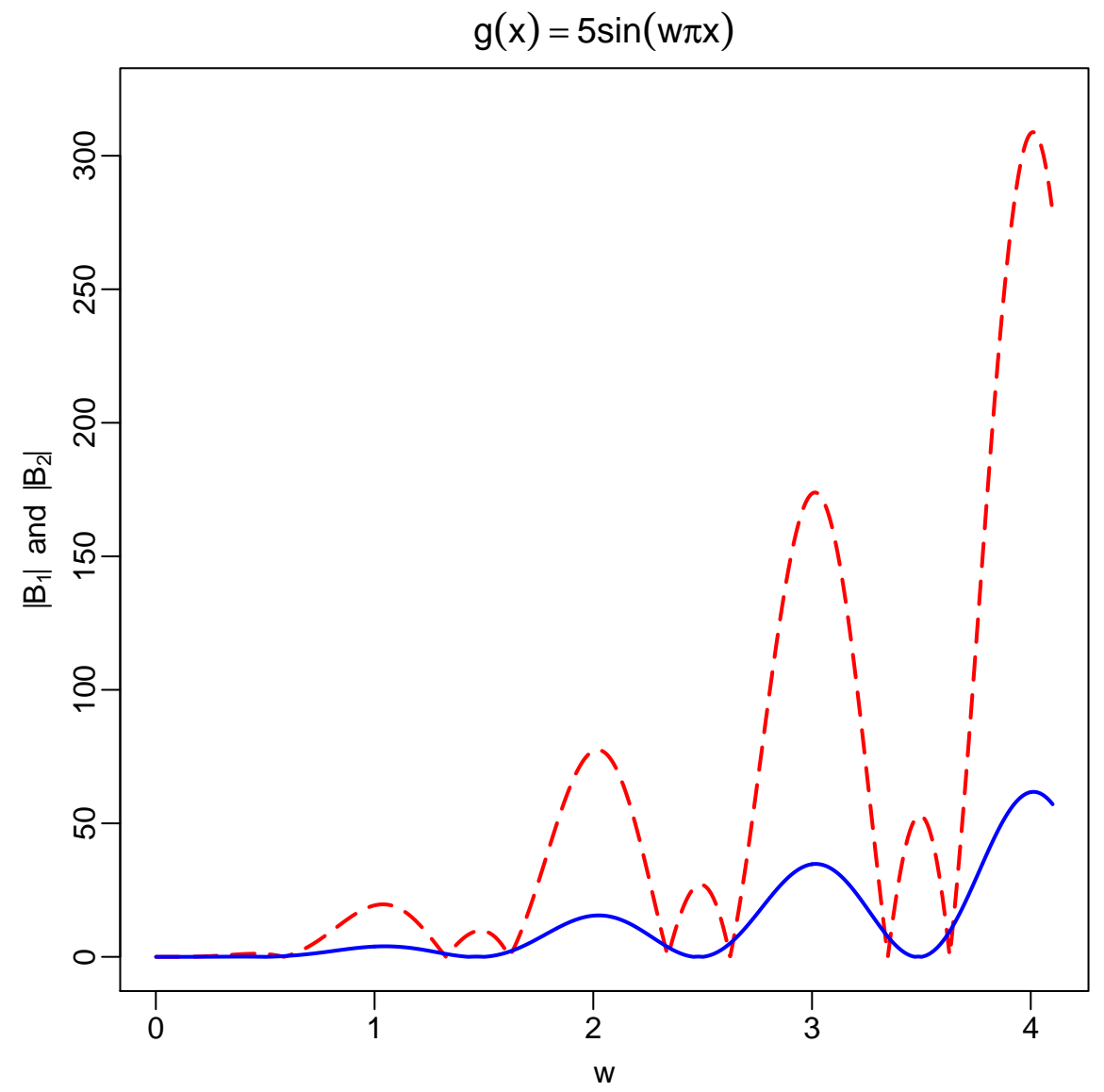

Figure S1: The absolute value of coefficients, $\left|B_{1}\right|$ and $\left|B_{2}\right|$, along with $w$ for $g(x)=$ $5 \sin (w \pi x)$. The blue solid line represents $\left|B_{1}\right|$, and the red dashed line represents $\left|B_{2}\right|$.

model as

$$
p_{i k}=\sigma^{2}+q_{i k}^{2}\left[g^{\prime}\left(x_{i}\right)\right]^{2}+\xi_{i k}, \quad(i, k) \in \mathcal{A}_{r}
$$

Consequently, by regressing $p_{i k}$ on $q_{i k}^{2}$ using least squares, we have the final estimator as $\hat{\sigma}^{2}(r, M)$. It can be verified that, with $M=m r / n, \hat{\sigma}^{2}(r, M)$ is equivalent to $\hat{\sigma}^{2}(r, m)$ for the equidistant design. Further, if $\left\{a_{i k j}\right\}$ satisfies $\sum_{j=0}^{r} a_{i k j}^{2}=1, \sum_{j=0}^{r} a_{i k j}=0$ and $\sum_{j=1}^{r} a_{i k j}\left(x_{i+k j}-x_{i}\right)^{K}=0$ for $K=1, \ldots, r-1$, we can show that $\hat{\sigma}_{\text {ord }}^{2}(r, M)$ is also equivalent to $\hat{\sigma}_{\text {ord }}^{2}(r, m)$. Further research might be needed to investigate the asymptotic properties under the unequally spaced design. 


\section{S5 Proofs of theoretical results}

In this section, we provide the technical proofs for the four theorems in Sections S1 and $\mathrm{S} 2$, respectively.

\section{Proof of Theorem S1}

First, we have $\sum_{k=1}^{m} b_{k} w_{k}=1$ and $\sum_{k=1}^{m} b_{k} w_{k} h_{k}=0$. (i) Let $g(x)=a x+b$, then

$$
\begin{aligned}
E\left(s_{k}(r)\right) & =\sigma^{2}+\frac{1}{n-k r} \sum_{i=1}^{n-k r}\left(\sum_{j=0}^{r} d_{j}\left(a \frac{(i+j k)}{n}+b\right)\right)^{2} \\
& =\sigma^{2}+\frac{1}{n-k r} \sum_{i=1}^{n-k r}\left(\frac{a k}{n} \sum_{j=0}^{r} d_{j} j\right)^{2} \\
& =\sigma^{2}+a^{2}\left(\sum_{j=0}^{r} d_{j} j\right)^{2} h_{k} .
\end{aligned}
$$

This leads to $E\left(\hat{\sigma}^{2}(r, m)\right)=\sigma^{2}+a^{2}\left(\sum_{j=0}^{r} d_{j} j\right)^{2} \sum_{k=1}^{m} b_{k} w_{k} h_{k}=\sigma^{2}$.

(ii) For the polynomial $g(x)$ with order up to $p \leq r-1$, let $g_{i}^{(p)}$ denote the $p$ th order derivative at design point $x_{i}$. Then

$$
\begin{aligned}
E\left(s_{k}(r)\right) & =\sigma^{2}+\frac{1}{n-k r} \sum_{i=1}^{n-k r}\left(\sum_{j=0}^{r} d_{j} g_{i+j k}\right)^{2} \\
& =\sigma^{2}+\frac{1}{n-k r} \sum_{i=1}^{n-k r}\left(d_{0} g_{i}+d_{1} \sum_{j=0}^{p} g_{i}^{(j)} \frac{(k / n)^{j}}{j !}+\cdots+d_{r} \sum_{j=0}^{p} g_{i}^{(j)} \frac{(j k / n)^{j}}{j !}\right)^{2} \\
& =\sigma^{2}+\frac{1}{n-k r} \sum_{i=1}^{n-k r}\left(C_{0} g_{i}+C_{1} g_{i}^{\prime} \frac{k}{n}+\cdots+C_{p} g_{i}^{(p)} \frac{k^{p}}{n^{p}}\right)^{2}
\end{aligned}
$$

where $C_{0}=\sum_{j=0}^{r} d_{j}$ and $C_{i}=\sum_{j=0}^{r} j^{i} d_{j} / i$ ! for $i=1, \ldots, r$. When $\left(d_{0}, \ldots, d_{r}\right)$ is the order- $r$ ordinary difference sequence, we have $C_{i}=0$ for $0 \leq i \leq p \leq r-1$. This results in $E\left(s_{k}(r)\right)=\sigma^{2}$ and, consequently, $E\left(\hat{\sigma}_{\text {ord }}^{2}(r, m)\right)=\sigma^{2}$.

\section{Proof of Theorem S2}

Note that

$$
E\left(s_{k}(r)\right)=\sigma^{2}+\frac{1}{n-k r} \sum_{i=1}^{n-k r}\left(\frac{1}{n} C_{1} g_{i}^{\prime} k+O\left(\frac{k^{2}}{n^{2}}\right)\right)^{2}=\sigma^{2}+C_{1}^{2} h_{k} \int_{0}^{1}\left[g^{\prime}(x)\right]^{2} d x+O\left(\frac{k^{3}}{n^{3}}\right) .
$$


We have

$$
\begin{aligned}
E\left(\hat{\sigma}^{2}(r, m)\right) & =\sum_{k=1}^{m} b_{k} w_{k}\left[\sigma^{2}+C_{1}^{2} h_{k} \int_{0}^{1}\left[g^{\prime}(x)\right]^{2} d x+O\left(\frac{k^{3}}{n^{3}}\right)\right] \\
& =\sigma^{2}+C_{1}^{2} \int_{0}^{1}\left[g^{\prime}(x)\right]^{2} d x \sum_{k=1}^{m} b_{k} w_{k} h_{k}+O\left(\frac{m^{3}}{n^{3}}\right) \\
& =\sigma^{2}+O\left(\frac{m^{3}}{n^{3}}\right) .
\end{aligned}
$$

For $s_{k}(r)$ with any difference sequence, with $k=o(n)$ and $k \rightarrow \infty$, we have

$$
\begin{aligned}
E\left[s_{k}(r)\right] & =\sigma^{2}+\frac{1}{n-r k} \sum_{i=1}^{n-r k}\left[C_{1} g^{\prime}\left(x_{i}\right) \frac{k}{n}+C_{2} g^{\prime \prime}\left(x_{i}\right) \frac{k^{2}}{n^{2}}+o\left(\frac{k^{2}}{n^{2}}\right)\right]^{2} \\
& =\sigma^{2}+C_{1}^{2} P_{A} \frac{k^{2}}{n^{2}}+2 C_{1} C_{2} P_{B} \frac{k^{3}}{n^{3}}+o\left(\frac{k^{3}}{n^{3}}\right),
\end{aligned}
$$

where $P_{A}=\sum_{i=1}^{n-r k}\left[g^{\prime}\left(x_{i}\right)\right]^{2} /(n-r k)$ and $P_{B}=\sum_{i=1}^{n-r k} g^{\prime}\left(x_{i}\right) g^{\prime \prime}\left(x_{i}\right) /(n-r k)$. For ease of notation, let $f(t)=\left[g^{\prime}(t)\right]^{2}$. Then,

$$
\begin{aligned}
P_{A}= & \frac{1}{n-r k} \sum_{i=1}^{n} f\left(x_{i}\right)-\frac{1}{n-r k} \sum_{i=n-r k+1}^{n} f\left(x_{i}\right) \\
= & \frac{n}{n-r k} \int_{0}^{1} f(t) d t-\frac{n}{n-r k} \sum_{i=1}^{n} \int_{x_{i-1}}^{x_{i}}\left(f(t)-f\left(x_{i}\right)\right) d t \\
& -\frac{n}{n-r k} \int_{x_{n-r k}}^{1} f(t) d t+\frac{n}{n-r k} \sum_{i=n-r k+1}^{n} \int_{x_{i-1}}^{x_{i}}\left(f(t)-f\left(x_{i}\right)\right) d t \\
\equiv & P_{1}-P_{2}-P_{3}+P_{4} .
\end{aligned}
$$

For $P_{1}$, we have $P_{1}=\int_{0}^{1} f(t) d t+(r k / n) \int_{0}^{1} f(t) d t+o(k / n)$. For $P_{2}$, we have

$$
\begin{aligned}
P_{2} & =\frac{n}{n-r k} \sum_{i=1}^{n} \int_{x_{i-1}}^{x_{i}}\left[f^{\prime}\left(x_{i}\right)\left(t-x_{i}\right)+O\left(\frac{1}{n^{2}}\right)\right] d t \\
& =\frac{n}{n-r k} \sum_{i=1}^{n}\left[-f^{\prime}\left(x_{i}\right) \frac{1}{2 n^{2}}+O\left(\frac{1}{n^{3}}\right)\right] d t \\
& =O\left(\frac{1}{n}\right) .
\end{aligned}
$$

For $P_{3}$, we let $F(s)=\int_{s}^{1} f(t) d t$. When $0<s<1$ is close to 1 , by Taylor expansion $F(s)=F(1)+F^{\prime}(1)(s-1)+O\left((1-s)^{2}\right)$ we have

$$
P_{3}=\frac{n}{n-r k} F\left(x_{n-r k}\right)=\frac{r k}{n} f(1)+O\left(\frac{k^{2}}{n^{2}}\right) .
$$


Finally, noting that $P_{4}=o(1 / n)$, we have

$$
P_{A}=\int_{0}^{1} f(t) d t+\frac{r k}{n}\left[\int_{0}^{1} f(t) d t-f(1)\right]+o\left(\frac{k}{n}\right) .
$$

Similarly as those for $P_{A}$, we can derive that

$$
P_{B}=\int_{0}^{1} g^{\prime}(t) g^{\prime \prime}(t) d t+o(1)=\left(\left[g^{\prime}(1)\right]^{2}-\left[g^{\prime}(0)\right]^{2}\right) / 2+o(1) .
$$

Substituting (S7) and (S8) into (S6), we have

$$
E\left(s_{k}(r)\right)=\sigma^{2}+\frac{k^{2}}{n^{2}} T_{1}+\frac{k^{3}}{n^{3}} T_{2}+o\left(\frac{k^{3}}{n^{3}}\right),
$$

where $T_{1}=C_{1}^{2} \int_{0}^{1}\left[g^{\prime}(t)\right]^{2} d t / 2$ and $T_{2}=C_{1}\left[r C_{1} \int_{0}^{1}\left[g^{\prime}(t)\right]^{2} d t-\left(r C_{1}-C_{2}\right)\left[g^{\prime}(1)\right]^{2}-C_{2}\left[g^{\prime}(0)\right]^{2}\right]$.

For the linear regression estimator $s_{k}(r), N=n m-r m(m+1) / 2$ and $w_{k}=(n-r k) / N$, we have

$$
\hat{\sigma}^{2}(r, m)=\sum_{k=1}^{m} w_{k} s_{k}(r)-\frac{\bar{h}_{w}}{\sum_{k=1}^{m} w_{k} h_{k}^{2}-\bar{h}_{w}^{2}} \sum_{k=1}^{m} w_{k}\left(h_{k}-\bar{h}_{w}\right) s_{k}(r)=Q_{1}-Q_{2} .
$$

Let $I_{t}=\sum_{k=1}^{m} k^{t}$ for $t=1, \ldots, 5$. For the first term, we have

$$
E\left(Q_{1}\right)=\sigma^{2}+\left(\frac{I_{2}}{N n}-\frac{r I_{3}}{N n^{2}}\right) T_{1}+\left(\frac{I_{3}}{N n^{2}}-\frac{r I_{4}}{N n^{3}}\right) T_{2}+o\left(\frac{m^{3}}{n^{3}}\right) .
$$

For the second term, we have

$$
\begin{aligned}
\bar{h}_{w} & =\frac{I_{2}}{N n}-\frac{r I_{3}}{N n^{2}}=\frac{m^{2}}{3 n^{2}}+o\left(\frac{m^{2}}{n^{2}}\right), \\
\sum_{k=1}^{m} w_{k} h_{k}^{2}-\bar{h}_{w}^{2} & =\frac{I_{4}}{N n^{3}}-\frac{r I_{5}}{N n^{4}}-\left(\frac{I_{2}}{N n}-\frac{r I_{3}}{N n^{2}}\right)^{2}=\frac{4 m^{4}}{45 n^{4}}+o\left(\frac{m^{4}}{n^{4}}\right), \\
\sum_{k=1}^{m} w_{k}\left(h_{k}-\bar{h}_{w}\right) E\left[s_{k}(r)\right] & =\left\{\frac{I_{4}}{N n^{3}}-\frac{r I_{5}}{N n^{4}}-\left(\frac{I_{2}}{N n}-\frac{r I_{3}}{N n^{2}}\right)^{2}\right\} T_{1} \\
& +\left\{\frac{I_{5}}{N n^{4}}-\frac{r I_{6}}{N n^{5}}-\left(\frac{I_{2}}{N n}-\frac{r I_{3}}{N n^{2}}\right)\left(\frac{I_{3}}{N n^{2}}-\frac{r I_{4}}{N n^{3}}\right)\right\} T_{2}+o\left(\frac{m^{5}}{n^{5}}\right) .
\end{aligned}
$$

This leads to the expectation of the second term as

$$
\begin{aligned}
E\left(Q_{2}\right) & =\frac{\frac{I_{2}}{N n}-\frac{r I_{3}}{N n^{2}}}{\frac{I_{4}}{N n^{3}}-\frac{r I_{5}}{N n^{4}}-\left(\frac{I_{2}}{N n}-\frac{r I_{3}}{N n^{2}}\right)^{2}}\left[\left\{\frac{I_{4}}{N n^{3}}-\frac{r I_{5}}{N n^{4}}-\left(\frac{I_{2}}{N n}-\frac{r I_{3}}{N n^{2}}\right)^{2}\right\} T_{1}\right. \\
& \left.+\left\{\frac{I_{5}}{N n^{4}}-\frac{r I_{6}}{N n^{5}}-\left(\frac{I_{2}}{N n}-\frac{r I_{3}}{N n^{2}}\right)\left(\frac{I_{3}}{N n^{2}}-\frac{r I_{4}}{N n^{3}}\right)\right\} T_{2}+o\left(\frac{m^{5}}{n^{5}}\right)\right] \\
& =\left(\frac{I_{2}}{N n}-\frac{r I_{3}}{N n^{2}}\right) T_{1}+C T_{2}+o\left(\frac{m^{3}}{n^{3}}\right)
\end{aligned}
$$


where

$$
\begin{aligned}
C & \left.=\frac{\frac{I_{5}}{N n^{4}}-\frac{r I_{6}}{N n^{5}}-\left(\frac{I_{2}}{N n}-\frac{r I_{3}}{N n^{2}}\right)\left(\frac{I_{3}}{N n^{2}}-\frac{r I_{4}}{N n^{3}}\right)}{\frac{I_{4}}{N n^{3}}-\frac{r I_{5}}{N n^{4}}-\left(\frac{I_{2}}{N n}-\frac{r I_{3}}{N n^{2}}\right)^{2}}-\frac{r I_{3}}{N n^{2}}\right) \\
& =\frac{\frac{m^{5}}{6 n^{5}}+o\left(\frac{m^{5}}{n^{5}}\right)-\left(\frac{m^{2}}{3 n^{2}}+o\left(\frac{m^{2}}{n^{2}}\right)\right)\left(\frac{m^{3}}{4 n^{3}}+o\left(\frac{m^{3}}{n^{3}}\right)\right)}{\frac{4 m^{4}}{45 n^{4}}+o\left(\frac{m^{4}}{n^{4}}\right)}\left(\frac{m^{2}}{3 n^{2}}+o\left(\frac{m^{2}}{n^{2}}\right)\right) \\
& =\frac{5 m^{3}}{16 n^{3}}+o\left(\frac{m^{3}}{n^{3}}\right) .
\end{aligned}
$$

Finally, by (S9) and (S10), we have $\operatorname{Bias}\left(\hat{\sigma}^{2}(r, m)\right)=-\left(m^{3} / 16 n^{3}\right) T_{2}+o\left(m^{3} / n^{3}\right)$ and this completes the derivation.

\section{Proof of Theorem S3}

For the ordinary difference sequence, we have $C_{1}=C_{2}=\cdots=C_{r-1}=0$ and $C_{r}=$ $\left(\begin{array}{c}2 r \\ r\end{array}\right)^{-1 / 2}$. Then,

$$
\begin{aligned}
E\left(s_{k}(r)\right) & =\sigma^{2}+\frac{1}{n-k r} \sum_{i=1}^{n-k r}\left(C_{1} g_{i}^{\prime} k / n+\cdots+C_{r} g_{i}^{(r)} k^{r} / n^{r}+o\left(k^{r} / n^{r}\right)\right)^{2} \\
& =\sigma^{2}+C_{r}^{2} \frac{k^{2 r}}{n^{2 r}} \int_{0}^{1}\left[g^{(r)}(x)\right]^{2} d x+o\left(\frac{k^{2 r}}{n^{2 r}}\right) .
\end{aligned}
$$

This leads to

$$
\begin{aligned}
E\left(\hat{\sigma}^{2}(r, m)\right) & =\sum_{k=1}^{m} b_{k} w_{k}\left[\sigma^{2}+C_{r}^{2} \frac{k^{2 r}}{n^{2 r}} \int_{0}^{1}\left[g^{(r)}(x)\right]^{2} d x+o\left(\frac{k^{2 r}}{n^{2 r}}\right)\right] \\
& =\sigma^{2}+C_{r}^{2} \int_{0}^{1}\left[g^{(r)}(x)\right]^{2} d x \sum_{k=1}^{m} b_{k} w_{k} \frac{k^{2 r}}{n^{2 r}}+o\left(\frac{m^{2 r}}{n^{2 r}}\right) \\
& =\sigma^{2}+\frac{3 C_{r}^{2}(1-r) m^{2 r}}{(2 r+1)(2 r+3) n^{2 r}} \int_{0}^{1}\left[g^{(r)}(x)\right]^{2} d x+o\left(\frac{m^{2 r}}{n^{2 r}}\right)
\end{aligned}
$$

where

$$
\begin{aligned}
\sum_{k=1}^{m} b_{k} w_{k} \frac{k^{2 r}}{n^{2 r}} & =\sum_{k=1}^{m} w_{k} \frac{k^{2 r}}{n^{2 r}}-\frac{\bar{h}_{w}}{\sum_{k=1}^{m} w_{k} h_{k}^{2}-\bar{h}_{w}^{2}} \sum_{k=1}^{m}\left(h_{k}-\bar{h}_{w}\right) \frac{k^{2 r}}{n^{2 r}} \\
& =\frac{1}{(2 r+1)} \frac{m^{2 r}}{n^{2 r}}(1+o(1))-\frac{15}{4}\left[\frac{1}{(2 r+3)}-\frac{1}{3(2 r+1)}\right] \frac{m^{2 r}}{n^{2 r}}(1+o(1)) \\
& =\frac{3(1-r)}{(2 r+1)(2 r+3)} \frac{m^{2 r}}{n^{2 r}}(1+o(1)) .
\end{aligned}
$$

This completes the derivation. 


\section{Proof of Theorem S4}

We first introduce three lemmas. Lemma S1 is used in the proof of Lemma S2, and Lemma S2 is used in the proof of Lemma S3. To save space, we only sketch the proof of Lemma S3.

Lemma S1. Assume that $m \rightarrow \infty$ and $m / n \rightarrow 0$. Then for $r=2$, we have

(a) $\sum_{k=1}^{m} h_{k}=\frac{m^{3}}{3 n^{2}}+\frac{m^{2}}{2 n^{2}}+o\left(\frac{m^{2}}{n^{2}}\right)$.

(b) $\bar{h}_{w}=\frac{1}{N n^{2}}\left[\frac{1}{3} n m^{3}+\frac{1}{2} n m^{2}-\frac{1}{2} m^{4}+o\left(n m^{2}\right)+o\left(m^{4}\right)\right]$.

(c) $\sum_{k=1}^{m} w_{k} h_{k}^{2}=\frac{1}{N n^{4}}\left[\frac{1}{5} n m^{5}+\frac{1}{2} n m^{4}-\frac{1}{3} m^{6}+o\left(n m^{4}\right)+o\left(m^{6}\right)\right]$.

(d) $\sum_{k=1}^{m} h_{k}^{2}=\frac{m^{5}}{5 n^{4}}+\frac{m^{4}}{2 n^{4}}+o\left(\frac{m^{4}}{n^{4}}\right)$.

(e) $\sum_{k=1}^{m} w_{k} h_{k}^{2}-\bar{h}_{w}^{2}=\frac{1}{N^{2} n^{4}}\left[\frac{4}{45} n^{2} m^{6}+\frac{1}{6} n^{2} m^{5}-\frac{1}{5} n m^{7}+o\left(n m^{7}\right)+o\left(n^{2} m^{5}\right)\right]$.

Lemma S2. Assume that $m \rightarrow \infty$ and $m / n \rightarrow 0$. Then for $r=2$, we have

(a) $\sum_{k=1}^{m} b_{k}=m-\frac{5 m^{2}}{8 n}+o\left(\frac{m^{2}}{n}\right)$.

(b) $\sum_{k=1}^{l-1} b_{k}=\frac{9}{4} l-\frac{5 l^{3}}{4 m^{2}}+o(l)+O(1), \quad 1 \leq l \leq m$.

(c) $\sum_{k=1}^{[(l-1) / 2]} b_{k}=\frac{9}{8} l-\frac{5 l^{3}}{32 m^{2}}+o(l)+O(1), \quad 1 \leq l \leq 2 m$.

(d) $\sum_{k=1}^{m} b_{k}^{2}=\frac{9}{4} m+o(m)$.

(e) $\sum_{k=1}^{m / 2} b_{k} b_{2 k}=\frac{9}{8} m+o(m)$.

(f) $\sum_{k=1}^{m} k b_{k}=O\left(m^{2}\right), \quad 1 \leq l \leq m$.

(g) $\sum_{k=1}^{l-1} k b_{k}=O\left(l^{2}\right), \quad 1 \leq l \leq m$.

(h) $\sum_{k=1}^{[(l-1) / 2]} k b_{k}=O\left(l^{2}\right), \quad 1 \leq l \leq 2 m$.

(i) $\sum_{k=1}^{m} k^{2} b_{k}=o\left(m^{3}\right)$.

Lemma S3. Under the same conditions as in Theorem S4, for $r=2$ we have

(a) $g^{T} D^{2} g=O\left(m^{5} / n^{2}\right)$. 
(b) $g^{T}(D \operatorname{diag}(D) u)=O\left(m^{4} / n\right)$.

(c) $\operatorname{tr}\left(D^{2}\right)=n m^{2}-\left[\frac{103}{56}-\frac{165}{448} d_{1}^{2}\left(1-d_{1}^{2}\right)\right] m^{3}+\left[\frac{9}{8}+\frac{9}{2}\left(d_{1}^{2}-\frac{1}{2}\right) d_{1}^{2}\right] m n+o\left(m^{3}+n m\right)$.

(d) $\operatorname{tr}\left\{\operatorname{diag}(D)^{2}\right\}=n m^{2}-\left[\frac{103}{56}-\frac{165}{448} d_{1}^{2}\left(1-d_{1}^{2}\right)\right] m^{3}+o\left(m^{3}\right)$.

Proof of Lemma S3. (a) Let $g_{i}=g\left(x_{i}\right), g_{i}^{\prime}=g^{\prime}\left(x_{i}\right)$ and $g_{i}^{\prime \prime}=g^{\prime \prime}\left(x_{i}\right)$ for $i=1, \ldots, n$. Noting that $D$ is symmetric, we have $g^{T} D^{2} g=g^{T} D^{T} D g=(D g)^{T} D g \triangleq p^{T} p$, where $p=D g=\left(p_{1}, p_{2}, \ldots, p_{n}\right)^{T}$. For $i \in[2 m+1, n-2 m]$, by Lemma S2 we have

$$
\begin{aligned}
p_{i} & =\left(d_{0} d_{1}+d_{1} d_{2}\right) \sum_{k=1}^{m} b_{k}\left(g_{i-k}+g_{i+k}-2 g_{i}\right)+d_{0} d_{2} \sum_{k=1}^{m} b_{k}\left(g_{i-2 k}+g_{i+2 k}-2 g_{i}\right) \\
& =\left(d_{0} d_{1}+d_{1} d_{2}\right) \sum_{k=1}^{m} b_{k}\left(\frac{k^{2}}{n^{2}} g_{i}^{\prime \prime}+o\left(\frac{k^{2}}{n^{2}}\right)\right)+4 d_{0} d_{2} \sum_{k=1}^{m} b_{k}\left(\frac{k^{2}}{n^{2}} g_{i}^{\prime \prime}+o\left(\frac{k^{2}}{n^{2}}\right)\right) \\
& =o\left(\frac{m^{3}}{n^{2}}\right) .
\end{aligned}
$$

Similarly, by Lemma S2 we can derive that Similarly, we can derive that $p_{i}=O\left(m^{2} / n\right)$ for $i \in[1, m], i \in[m+1,2 m], i \in[n-2 m+1, n-m]$, and $i \in[n-m+1, n]$, respectively. This leads to $g^{T} D^{2} g=\sum_{i=1}^{2 m} p_{i}^{2}+\sum_{i=2 m+1}^{n-2 m} p_{i}^{2}+\sum_{i=n-2 m+1}^{n} p_{i}^{2}=O\left(m^{5} / n^{2}\right)$.

(b) By (a), (b) and (c) in Lemma S2, we have $g^{T}(D \operatorname{diag}(D) u)=p^{T} \operatorname{diag}(D) u=$ $\left(\sum_{i=1}^{m}+\sum_{i=m+1}^{2 m}+\sum_{i=1+2 m}^{n-2 m}+\sum_{i=n-2 m+1}^{n-m}+\sum_{i=n-m+1}^{n}\right) p_{i} d_{i i}=O\left(m^{4} / n\right)$.

(c) By some simple calculation, we have

$$
\begin{aligned}
\sum_{i=1}^{m} \sum_{j=1}^{n} d_{i j}^{2} & =\left(d_{0}^{4}+\frac{11}{14} d_{1}^{4}+\frac{2545}{7168} d_{2}^{4}+\frac{13}{8} d_{0}^{2} d_{1}^{2}+\frac{233}{224} d_{1}^{2} d_{2}^{2}+\frac{67}{64} d_{0}^{2} d_{2}^{2}\right) m^{3}+o\left(m^{3}\right), \\
\sum_{i=m+1}^{2 m} \sum_{j=1}^{n} d_{i j}^{2} & =\left(d_{0}^{4}+d_{1}^{4}+\frac{8719}{7168} d_{2}^{4}+2 d_{0}^{2} d_{1}^{2}+\frac{141}{64} d_{1}^{2} d_{2}^{2}+\frac{141}{64} d_{0}^{2} d_{2}^{2}\right) m^{3}+o\left(m^{3}\right), \\
\sum_{i=2 m+1}^{n-2 m} \sum_{j=1}^{n} d_{i j}^{2} & =n m^{2}-\frac{21}{4} m^{3}+\left(\frac{9}{8}+\frac{9}{2} d_{0} d_{1}^{2} d_{2}\right) n m+o(n m)+o\left(m^{3}\right), \\
\sum_{i=n-2 m+1}^{n-m} \sum_{j=1}^{n} d_{i j}^{2} & =\left(\frac{8719}{7168} d_{0}^{4}+d_{1}^{4}+d_{2}^{4}+\frac{141}{64} d_{0}^{2} d_{1}^{2}+2 d_{1}^{2} d_{2}^{2}+\frac{141}{64} d_{0}^{2} d_{2}^{2}\right) m^{3}+o\left(m^{3}\right), \\
\sum_{i=n-m+1}^{n} \sum_{j=1}^{n} d_{i j}^{2} & =\left(\frac{2545}{7168} d_{0}^{4}+\frac{11}{14} d_{1}^{4}+d_{2}^{4}+\frac{233}{224} d_{0}^{2} d_{1}^{2}+\frac{13}{8} d_{1}^{2} d_{2}^{2}+\frac{67}{64} d_{0}^{2} d_{2}^{2}\right) m^{3}+o\left(m^{3}\right) .
\end{aligned}
$$


This leads to

$$
\begin{aligned}
\operatorname{tr}\left(D^{2}\right) & =\left(\sum_{i=1}^{m} \sum_{j=1}^{n}+\sum_{i=m+1}^{2 m} \sum_{j=1}^{n}+\sum_{i=2 m+1}^{n-2 m} \sum_{j=1}^{n}+\sum_{i=n-2 m+1}^{n-m} \sum_{j=1}^{n}+\sum_{i=n-m+1}^{n} \sum_{j=1}^{n}\right) d_{i j}^{2} \\
& =n m^{2}-\left[\frac{103}{56}-\frac{165}{448} d_{1}^{2}\left(1-d_{1}^{2}\right)\right] m^{3}+\left[\frac{9}{8}+\frac{9}{2}\left(d_{1}^{2}-\frac{1}{2}\right) d_{1}^{2}\right] m n+o\left(m^{3}+n m\right) .
\end{aligned}
$$

(d) Similarly as in the proof of part (c), we have

$$
\begin{aligned}
\operatorname{tr}\left\{\operatorname{diag}(D)^{2}\right\} & =\left(\sum_{i=1}^{m}+\sum_{i=m+1}^{2 m}+\sum_{i=1+2 m}^{n-2 m}+\sum_{i=n-2 m+1}^{n-m}+\sum_{i=n-m+1}^{n}\right) d_{i i}^{2} \\
& =n m^{2}-\left[\frac{103}{56}-\frac{165}{448} d_{1}^{2}\left(1-d_{1}^{2}\right)\right] m^{3}+o\left(m^{3}\right) .
\end{aligned}
$$

Proof of Theorem S4. By Lemma S3, formula (S5) and the fact that $\operatorname{tr}(D)=N$, we have the variance of $\hat{\sigma}^{2}(2, m)$ as

$$
\begin{aligned}
\operatorname{var}\left(\hat{\sigma}^{2}(2, m)\right)= & \frac{1}{N^{2}}\left\{O\left(\frac{m^{5}}{n^{2}}\right)+O\left(\frac{m^{4}}{n}\right)\right. \\
& +\left(\operatorname{var}\left(\varepsilon^{2}\right)-2 \sigma^{4}\right)\left[n m^{2}-\left[\frac{103}{56}-\frac{165}{448} d_{1}^{2}\left(1-d_{1}^{2}\right)\right] m^{3}+o\left(m^{3}\right)\right] \\
& +2 \sigma^{4}\left[n m^{2}-\left[\frac{103}{56}-\frac{165}{448} d_{1}^{2}\left(1-d_{1}^{2}\right)\right] m^{3}\right. \\
& \left.\left.+\left[\frac{9}{8}+\frac{9}{2}\left(d_{1}^{2}-\frac{1}{2}\right) d_{1}^{2}\right] m n+o\left(m^{3}+n m\right)\right]\right\} \\
= & \frac{1}{n} \operatorname{var}\left(\varepsilon^{2}\right)+A_{1} \frac{\sigma^{4}}{m n}+A_{2} \frac{m}{n^{2}} \operatorname{var}\left(\varepsilon^{2}\right)+o\left(\frac{1}{n m}\right)+o\left(\frac{m}{n^{2}}\right),
\end{aligned}
$$

where $A_{1}$ are $A_{2}$ are defined in Theorem S4. This proves the theorem.

\section{References}

Dette, H., Munk, A. and Wagner, T. (1998). Estimating the variance in nonparametric regression - what is a reasonable choice?, Journal of the Royal Statistical Society, Series B 60: 751-764.

Hall, P., Kay, J. W. and Titterington, D. M. (1990). Asymptotically optimal differencebased estimation of variance in nonparametric regression, Biometrika 77: 521-528.

Seifert, B., Gasser, T. and Wolf, A. (1993). Nonparametric estimation of residual variance revisited, Biometrika 80: 373-383. 
Tong, T., Ma, Y. and Wang, Y. (2013). Optimal variance estimation without estimating the mean function, Bernoulli 19: 1839-1854.

Tong, T. and Wang, Y. (2005). Estimating residual variance in nonparametric regression using least squares, Biometrika 92: 821-830. 